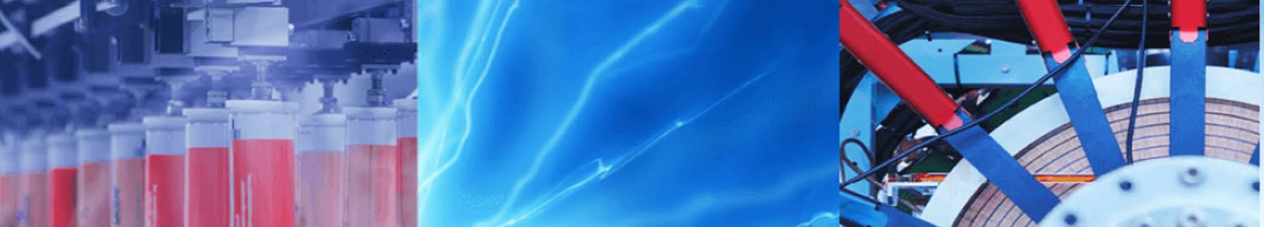

Research Article

\title{
An autonomous real-time detector system for radionuclide monitoring in drinking water systems
}

\author{
Jorrit Drinhaus $^{1}$ (1) . Alexander Harstrick ${ }^{1}$. Bastian Breustedt ${ }^{1}$
}

Received: 5 September 2020 / Accepted: 7 January 2021 / Published online: 27 January 2021

(C) The Author(s) 2021 OPEN

\begin{abstract}
For monitoring radioactive contamination of water systems due to nuclear accidents or terrorist attacks, there is need of an in situ online measurement to assess alpha-, beta- and gamma-emitting radionuclide contaminants quickly and accurately. However, there is no well-established online monitoring system for permanent surveillance of drinking water systems. Therefore, a real-time measurement system was developed based on the readout of plastic scintillator sheets by a photomultiplier. Direct contact between scintillator and streaming water allows for the detection of both long- and short-ranged particles. Using a passive cooling system, detection limits of $141 \mathrm{~Bq} / \mathrm{L}$ for ${ }^{241} \mathrm{Am}, 20 \mathrm{~Bq} / \mathrm{L}$ for ${ }^{60} \mathrm{Co}$ and $17 \mathrm{~Bq} / \mathrm{L}$ for ${ }^{137} \mathrm{Cs}$ were achieved with measurements of $60 \mathrm{~s}$ counting time. The system has been designed to be operated safely without deep knowledge of radiation measurement technology. The detector can be connected as a bypass to a water system and be operated in a continuous online survey mode, making it applicable as an early warning system.
\end{abstract}

Keywords Monitoring $\cdot$ Drinking water $\cdot$ Radionuclides $\cdot$ Scintillation counting $\cdot$ Emergency situation

\section{Introduction}

The ingestion of radionuclides is one pathway for human radiation exposure and can therefore increase cancer risk [1]. Hence, it is important for public health to assure a safe water supply. One potential risk is that drinking water systems are vulnerable to contamination with radioactive material, either by accidents or malevolent acts. Methods applied in routine monitoring of water systems (like regular sampling with subsequent laboratory analysis) cannot detect short-time contaminations and can therefore not prevent a resulting radiation exposure of the population [2]. To overcome this problem, embedded in the TransAqua competence network [3], a real-time measurement system based on a scintillation detector was developed that can detect contaminations with radionuclides emitting alpha, beta or gamma radiation. An automatic analysis will trigger a warning issued if given thresholds of contamination are exceeded. The system is only intended to detect the contamination. It is not required that the system is able to identify and quantify the nuclides. This analysis needs to be done using standardised techniques in a laboratory. The latter will allow nuclide identification and more precise information about respective activity concentrations. The aim of this detector is to allow for fast response (i.e. within minutes) in case of a radiological emergency. Our approach differs from comparable detector developments that are done at the moment [4] in its simplicity and, therefore, lower cost. Maximum permitted levels of radioactive contamination in liquid food defined in [5] are used as reference values in the design of the system.

$\checkmark$ Jorrit Drinhaus, drinhaus.j@gmail.com | ${ }^{1}$ Karlsruhe Institute of Technology (KIT), Safety and Environment (SUM), Hermann-von-Helmholtz-Platz 1, 76344 Eggenstein-Leopoldshafen, Germany. 
Fig. 1 Simulated detection efficiency normalised to the smallest simulated distance

Fig. 2 Simulated count rate normalised to the smallest simulated distance
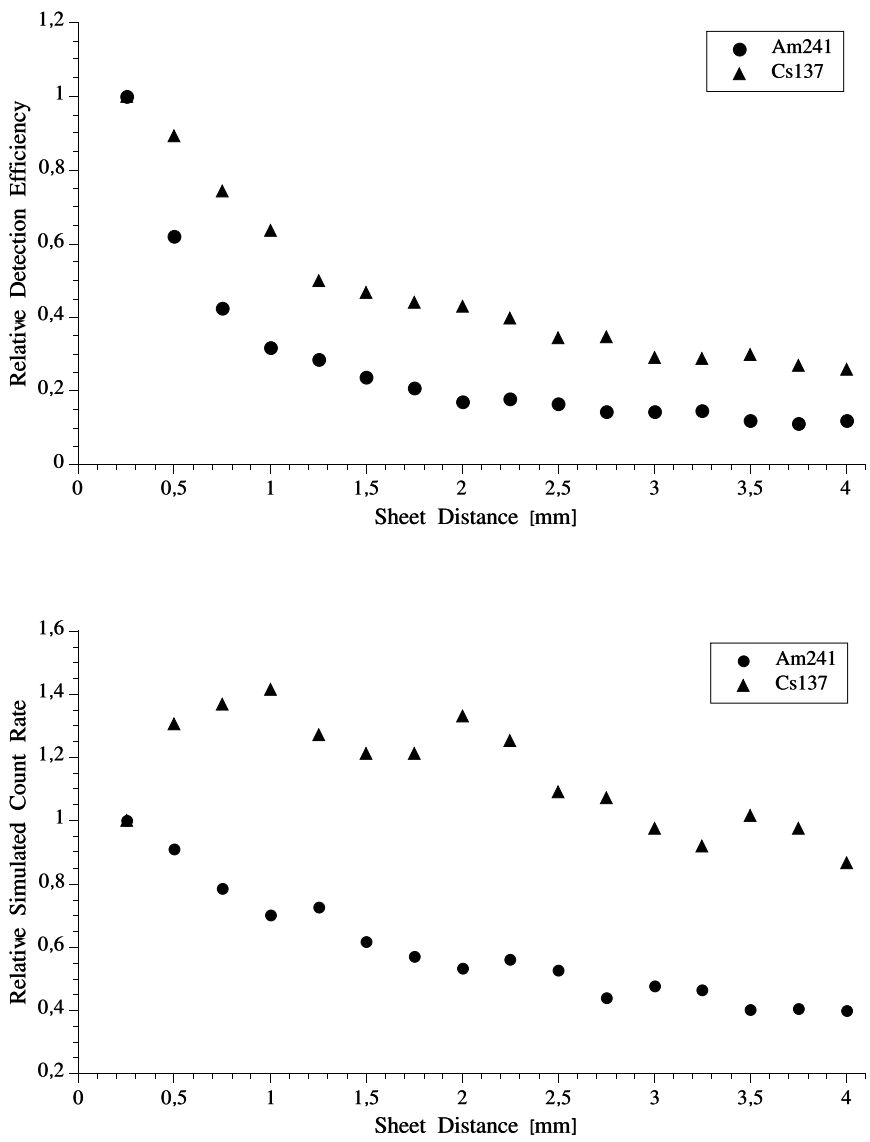

\section{Materials and methods}

\subsection{Monte Carlo simulations}

Detector material plastic scintillator sheets have been selected. This is due to the fact that plastic scintillators are relatively cheap, easy to handle and non-hygroscopic. This allows for a large surface inside the water volume to be measured needed to reliably detect shortranged particles like alpha particles and low-energy beta particles. In order to find an appropriate detector setup, Monte Carlo simulations were performed using the GEANT4-based GAMOS framework [6]. The investigation was started with simulations of different setups of radioactive sources and an existing $\mathrm{Nal}$ scintillation detector [7]. The simulation results were in accordance with the respective measurements, validating the used tool as being suitable simulation framework for this application. After that, other simple and more complex structures have been investigated in regard to their suitability as a detector in streaming water. Judging by the results of these simulations, also taking into account certain physical constraints, a stack of scintillating sheets appeared to be a reasonable geometry for this application. With the aim of finding an efficient detector setup, simulations with different sheet thicknesses and distances between the sheets have been conducted. The evaluation of the simulated data had to be done considering practical constraints like ensuring stability and a constant water flow between the scintillating sheets. Figure 1 shows the results for the efficiency, i.e. the relative number of scintillation photons reaching the photomultiplier per decay. This efficiency decreases with increasing distance between the sheets for both ${ }^{137} \mathrm{Cs}$ and ${ }^{241} \mathrm{Am}$. The effect originates from both coverage of the solid angle and mean distance to the scintillator. Due to the short range of alpha particles in water, the effect is stronger for ${ }^{241} \mathrm{Am}$. The relative efficiency values neglect the increase in the total activity in the measuring chamber for larger distances between the scintillating sheets. Figure 2 shows the simulated count rate which is the product of efficiency and net water volume, therefore taking into account the increase in activity being measured inside the detector. For ${ }^{137} \mathrm{Cs}$, the increasing water volume compensates for the decreasing efficiency, while for ${ }^{241} \mathrm{Am}$, the overall count rate decreases. Yet the distance between the scintillating sheets was set to $2 \mathrm{~mm}$ to ensure continuous water flow between and around the sheets. Analogously, the sheet thickness was set to 
Fig. 3 Layout of the detector prototype shown as a section of the xz-plane (left) and of the yz-plane (right). Water enters through an inlet at the bottom, traverses the detection volume and exits via a spillover

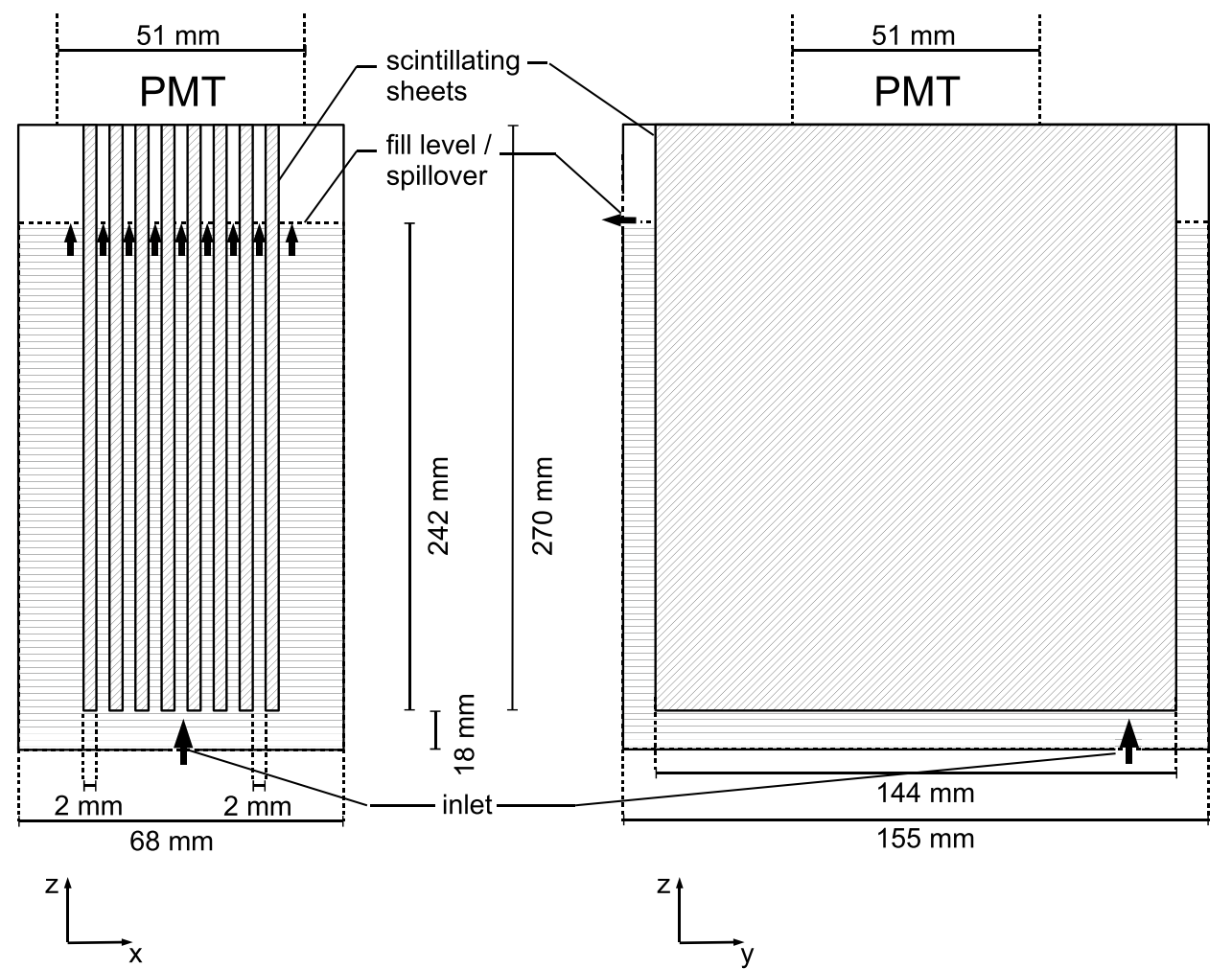

$2 \mathrm{~mm}$ as well considering scintillation and light guide efficiency and mechanical stability.

\subsection{Detector setup}

The detector prototype consists of a stack of 8 scintillating sheets (Epic-Crystal polystyrene plastic scintillator [8]). The dimensions of each sheet are $2 \times 144 \times 270 \mathrm{~mm}^{3}(\mathrm{WxLXH})$, having the upper $28 \mathrm{~mm}$ of the scintillating sheets outside of the measuring volume, thus leading to an effective surface of almost $5700 \mathrm{~cm}^{2}$ (see Fig. 3). The basis of $155 \times 68 \mathrm{~mm}^{2}$ and the fill level of $26 \mathrm{~cm}$ result in a net water volume in the measuring chamber of $2.2 \mathrm{~L}$. Water enters the measuring chamber via an inlet at the bottom. It traverses the scintillating sheets and leaves the detector volume via the spillover. In the loop used for testing the device, the water is entering a reservoir. From there, it is led back into the measuring chamber using a peristaltic pump. The inlet at the bottom combined with the spillover assures a constant filling of the measuring chamber. The system with inlet and spillover outlet is designed to be connected as bypass to a flowing water system. Light generated by the scintillating sheets is detected by a photomultiplier (Hamamatsu R2154-02 [9]) which is coupled to a Canberra Osprey tube base [10] containing power supply, preamplifier and multichannel analyser. Power supply for the tube base is done via the USB connection of the Osprey module. For the current setup, we refrained from using a light guide in order to first evaluate whether it is necessary in order for the detector system to fulfil its purpose. Rapid prototyping was used to manufacture the lightproof housing with a 3D printer (Ultimaker 2 [11]) using ABS polymer. A modular design allows for fast improvements by modifying only relevant parts instead of reprinting the whole detector housing. The photomultiplier is helically encased by a flexible tube used as a cooling system. Cooling water can be supplied either externally with a well-defined temperature, or the extracted water that is to be measured can be used. This makes it convenient to use and unsusceptible to malfunctions, however, at the cost of control of the cooling temperature.

\subsection{Characteristics of the system}

The light collection efficiency of the system is rather poor, resulting in a reduced efficiency that could be improved. One reason for this is the black surfaces of the casing that have a low reflectivity. Using reflective paint or mirror foil for the inner surfaces would on the one hand increase light collection and thus efficiency, but on the other hand, this would lead to a strong dependency of the light collection on the transparency of the water. This would make the system vulnerable to any increase in opacity and thus less universally applicable. An application with natural water, e.g. with river water that changes its opacity, would 
become less stable. Because of this, we decided not to further increase the reflectivity.

\subsection{Rapid prototyping}

The usage of a 3D printer facilitated the development of the housing by making it faster, cheaper and more versatile than using preformed parts or manufacturing it in a workshop. However, the printed parts were in need of a post-manufacture treatment with acetone, dissolving the printed surface of the ABS material and leaving a smooth surface after evaporating. This not only covers occurring gaps between printed layers and assures a waterproof and plain surface to avoid contamination due to accumulation of radionuclides but also strengthens the binding between the printed layers and therefore improves the overall stability and durability of the printed parts. The treatment was done using a spray flask in order to precisely dispense the acetone as excessive usage thereof can dissolve the supporting structure and therefore harm the printed parts.

\subsection{Data readout and analysis}

Communication with the Osprey tube base can be done via USB or Ethernet connection. To control and read out the tube base, dedicated software including a graphical user interface (GUI) was written in $\mathrm{C}++$ using the provided software development kit [10]. This software allows for continuous measurements as well as measurement series with alternating parameters and therefore meets the needs of both application and development. The GUI offers control over parameters such as preamplifier gain, photomultiplier voltage and measurement time. Furthermore, the software provides data storage and a visualisation of the count rates over time in a graph. The extracted data can be used for time series analysis. Due to the limited energy resolution of the plastic scintillator and the fact that the Osprey system does not offer a time resolved spectrum, the detector does not offer nuclide identification and count rates are determined solely by integrating the spectra (i.e. net sum in predefined region of interest) acquired by the multichannel analyser. Thresholds for the count rate as well as for the change in the count rate over time are possible triggers for an alarm.

\subsection{Characteristic limits}

The sensitivity of a detector is described by its characteristic limits, the decision threshold $y^{*}$ and the detection limit $y^{\#}$. The decision threshold indicates the measurement result that affirms the presence of the measured element. There is a probability $\alpha$ of a result above the decision threshold $y^{*}$ in measurements without the presence of the measured element. The detection limit $y^{\#}$ indicates the concentration of the measured element that would lead to a result below the decision threshold $y^{*}$ with a probability of $\beta$. All calculations that are shown in this study were done using $\alpha=\beta=5 \%$. Due to the influence of type and energy of the emitted radiation, characteristic limits are dependent on the radionuclide that is being measured. We will determine the characteristic limits of the presented detector systems for three different radionuclides representing possible contaminants in order to evaluate the suitability of the presented detector system as a trigger system for fast responses on radiological contaminations of drinking water systems. The determination of characteristic limits is done according to ISO 11929 [12]. As described in [13], $y^{*}$ and $y^{\#}$ for integral counting of alpha/beta activity are

$y^{*}=\frac{w}{t_{g}} k \sqrt{\frac{t_{g}}{t_{0}}\left(\frac{t_{g}}{t_{0}}+1\right) n_{0}}$

and

$y^{\#}=\frac{w}{t_{g}} \frac{k^{2}+2 k \sqrt{\frac{t_{g}}{t_{0}}\left(\frac{t_{g}}{t_{0}}+1\right) n_{0}}}{1-k^{2}\left(\frac{u(w)}{w}\right)^{2}}$

which can be rewritten to

$y^{*}=k w \sqrt{r_{0}\left(\frac{1}{t_{g}}+\frac{1}{t_{0}}\right)}$

and

$y^{\#}=\frac{2 y^{*}+k^{2} w / t_{g}}{1-k^{2}\left(\frac{u(w)}{w}\right)^{2}}$

with

$y^{*}: \quad$ Decision threshold in Bq/kg

$k$ : $\quad$ Quantile of the standard normal distribution, $\alpha=5$ $\%$

w: $\quad$ Conversion factor in $(\mathrm{Bq} / \mathrm{kg}) / \mathrm{s}^{-1}$

$t_{0}: \quad$ Blank measurement time

$t_{g}: \quad$ Sample measurement time

$n_{0}$ : Blank counts

$y^{\#}: \quad$ Detection limit

$u(w)$ : Combined standard uncertainty of $w$

$r_{0}: \quad$ Blank count rate $\left(n_{0} / t_{0}\right)$

\subsection{Calibration measurements}

In order to calculate detection limits, measurements of the blank count rate as well as measurements with reference solutions were performed. All measurements 
Table 1 Radionuclides used for calibration measurements

\begin{tabular}{llll}
\hline Nuclide & $\begin{array}{l}\text { alpha energy } \\
{[\mathrm{MeV}]}\end{array}$ & $\begin{array}{l}\text { beta max. energy } \\
{[\mathrm{MeV}]}\end{array}$ & $\begin{array}{l}\text { gamma } \\
\text { energy } \\
{[\mathrm{MeV}]}\end{array}$ \\
\hline${ }^{60} \mathrm{Co}$ & - & 0.317 & $1.17,1.33$ \\
${ }^{137} \mathrm{Cs}$ & - & $0.514,1.18$ & 0.662 \\
${ }^{241} \mathrm{Am}$ & 5.58 & - & 0.0595 \\
\hline
\end{tabular}

Shown are only the main energies. Data taken from Laboratoire National Henri Becquerel [14]

Fig. 4 Voltage dependence of the blank count rate $r_{0}$ with cooling system at $10^{\circ} \mathrm{C}$ (triangles) and without cooling system (circles) contaminations with other radionuclides of more than $1 \%$ of the reference activity.

\section{Results}

\subsection{Detection limits}

Optimal settings for preamplifier gain and photomultiplier voltage were determined in measurement series

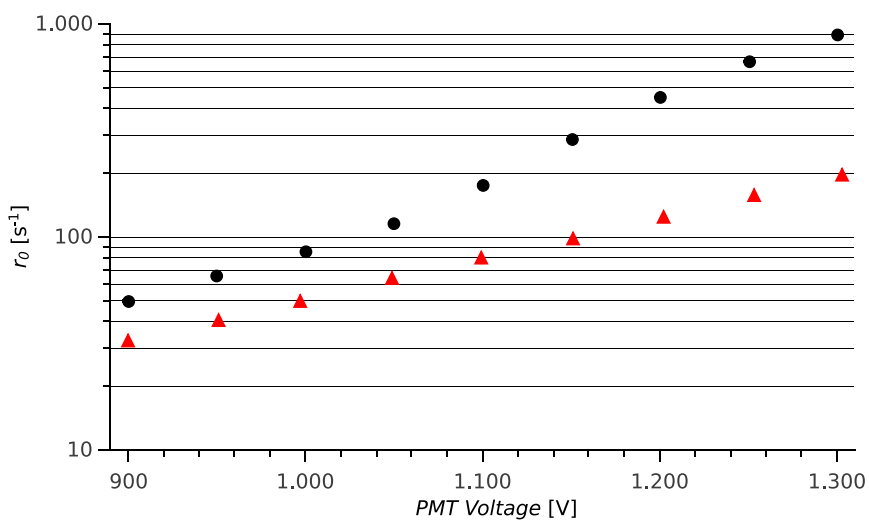

with altering parameters. Due to the poor light collection efficiency, the voltages required are rather high, resulting in high levels of dark currents which increase the background count rate (up to $669 \mathrm{~s}^{-1}$ at a voltage of $1250 \mathrm{~V}$, Fig. 4). Using the cooling system running with water at $10^{\circ} \mathrm{C}$ (typical temperature in water systems), we were able to reduce the background count rate at $10^{\circ} \mathrm{C}$ to $158 \mathrm{~s}^{-1}$.

As Fig. 5 indicates, optimal (with regard to the detection limit) photomultiplier voltages vary, depending on the measured radionuclides. This is due to different conversion factors $w$ resulting from different radiation types and energies. With increasing photomultiplier voltage, the blank count rate $r_{0}$ rises, while the conversion factor $w$ decreases (Figs. 4, 7), leading to an optimal decision threshold where the product is minimal (Fig. 5). Since alpha emitters are the most dose-relevant nuclides and are the hardest to detect, the optimal voltage for detecting alpha particles was chosen as reference voltage for the following measurements. Using the cooling system at $10{ }^{\circ} \mathrm{C}$ resulted in a shift in the optimal voltage from 1200 to $1250 \mathrm{~V}$ (Fig. 6) due to the fact that with the cooling system, the negative impact of the rising background count rate is not as severe as it is without cooling system. As can be seen in Tables 2 and 3 , by using the cooling system at $10^{\circ} \mathrm{C}$ we were able to reduce the detection limit $y^{\#}$ roughly by half, depending on the radionuclide. 
Fig. 5 Voltage dependence of the detection limit $y^{\#}$ without cooling system
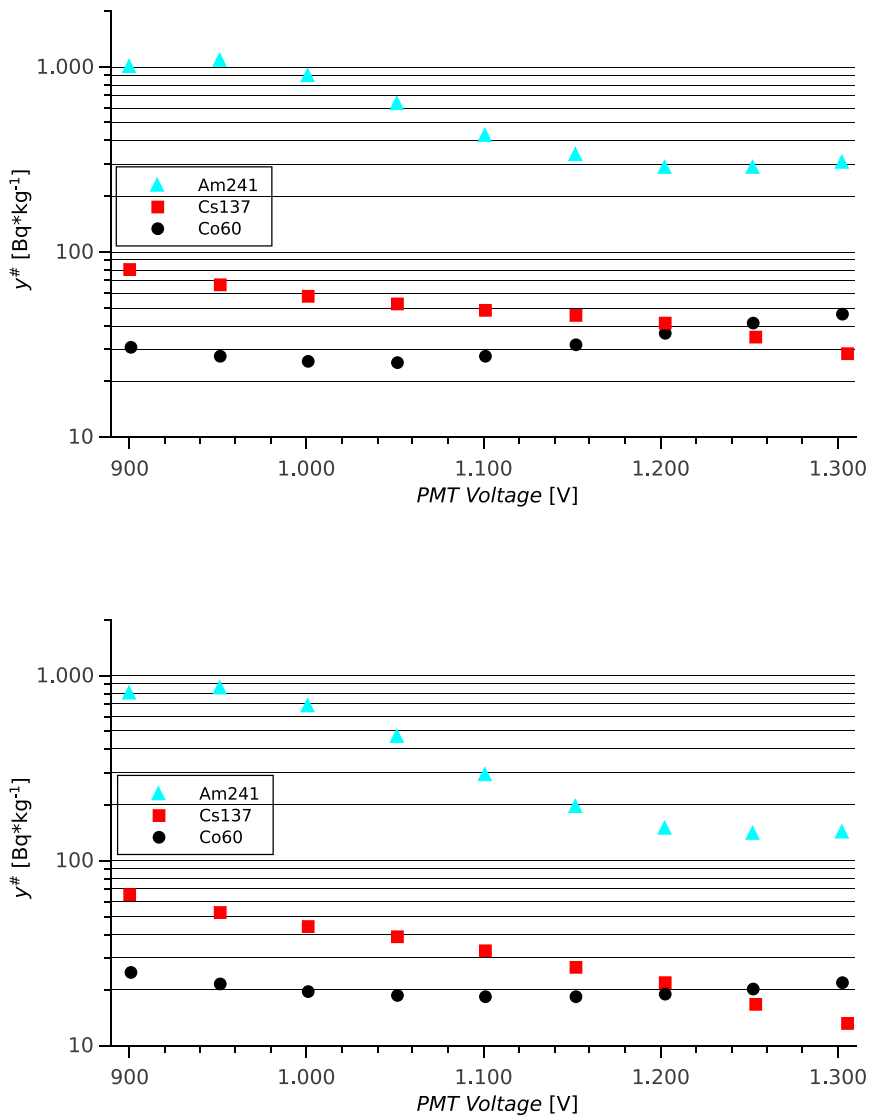

Fig. 6 Voltage dependence of the detection limit $y^{\#}$ with cooling system at $10^{\circ} \mathrm{C}$
Fig. 7 Voltage dependence of the conversion factor $w$

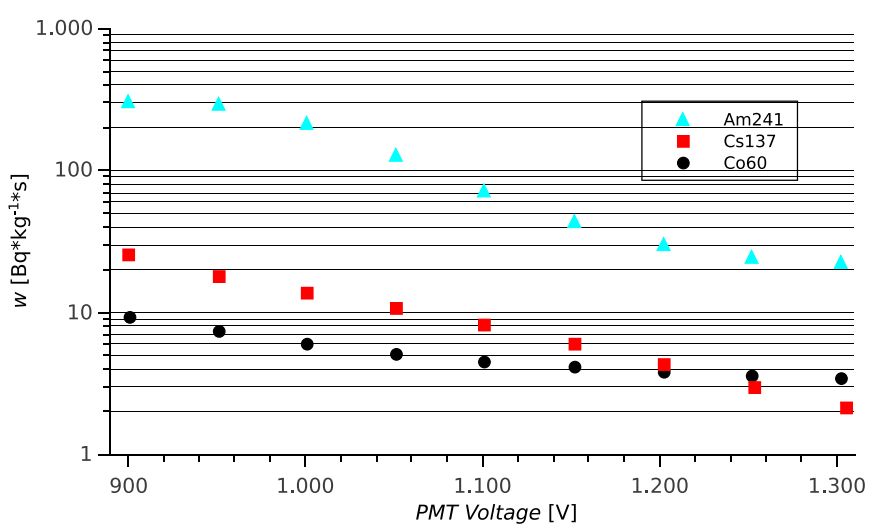

Table 3 Characteristic limits for different radionuclides at $U=1250 \mathrm{~V}$ with $t_{0}=600 \mathrm{~s}, t_{g}=60 \mathrm{~s}$ and $k=1.645$ using the cooling system at $10^{\circ} \mathrm{C}$ with a background count rate of $r_{0}=158 \mathrm{~s}^{-1}$

Nuclide $\quad w\left[(\mathrm{~Bq} / \mathrm{kg})^{*} \mathrm{~s}\right] \quad y^{*}[\mathrm{~Bq} / \mathrm{kg}] \quad y^{\#[\mathrm{~Bq} / \mathrm{kg}]}$ Max. perm. Level $[\mathrm{Bq} /$ $\mathrm{kg}]$

\begin{tabular}{lllll}
\hline${ }^{60} \mathrm{Co}$ & 3.59 & 10.1 & 20.4 & 1000 \\
${ }^{137} \mathrm{Cs}$ & 3.00 & 8.39 & 17.0 & 1000 \\
${ }^{241} \mathrm{Am}$ & 24.9 & 69.7 & 141 & 20 \\
\hline
\end{tabular}

\section{SN Applied Sciences}

Max. perm. $\mathrm{kg}]$

\begin{tabular}{lllll}
\hline${ }^{60} \mathrm{Co}$ & 3.82 & 18.1 & 36.7 & 1000 \\
${ }^{137} \mathrm{Cs}$ & 4.38 & 20.8 & 41.9 & 1000 \\
${ }^{241} \mathrm{Am}$ & 30.1 & 143 & 289 & 20 \\
\hline
\end{tabular}


Table 4 Effective doses resulting from an ingestion of $0.5 / 2 \mathrm{~L}$ (infant/adult) with an activity concentration at the detection limit $y^{\#}$ using the cooling system

\begin{tabular}{lllll}
\hline Nuclide & $e_{\text {infant }}$ & $e_{\text {adult }}$ & $\begin{array}{l}D_{\text {inf }}(0.5 \mathrm{~kg} \\
\left.@ y^{\#}\right)[\mathrm{mSv}]\end{array}$ & $\begin{array}{l}D_{\mathrm{ad}}(2 \mathrm{~kg} \\
\left.@ y^{\#}\right) \\
{[\mathrm{mSv}]}\end{array}$ \\
\hline${ }^{60} \mathrm{Co}$ & $5.4 \mathrm{E}-08$ & $3.4 \mathrm{E}-09$ & 0.00055 & 0.00014 \\
${ }^{137} \mathrm{Cs}$ & $2.1 \mathrm{E}-08$ & $1.3 \mathrm{E}-08$ & 0.00018 & 0.00044 \\
${ }^{241} \mathrm{Am}$ & $3.7 \mathrm{E}-06$ & $2.0 \mathrm{E}-07$ & 0.26 & 0.056 \\
\hline
\end{tabular}

\subsection{Detection capabilities in early response}

The results of the calibration measurements are presented in Table 2 (without cooling system) and Table 3 (with cooling system). It shows the conversion factors and the calculated decision thresholds and detection limits for measurement times of $t_{0}=600 \mathrm{~s}$ and $t_{g}=60 \mathrm{~s}$ for $k=1.645$ $(\alpha=\beta=0.05)$. The corresponding maximum permitted levels [5] are added for comparison. It has to be kept in mind that these are the maximum permitted levels for permanent consumption and, therefore, do not have to be achieved in fast measurements. These levels are based on a reference level of $1 \mathrm{mSv} / \mathrm{a}$ under the assumption of a consumption of $10 \%$ contaminated food. (Drinking water is classified as liquid food.) It can be seen that for the beta and gamma nuclides, detection limits are below the maximum permitted levels for measurement times of $60 \mathrm{~s}$. For the alpha emitter ${ }^{241} \mathrm{Am}$, it takes blank and sample measurement times of $2 \mathrm{~h}$ each to meet the maximum permitted level. These longer measurement times still allow for long-term surveillance, since the maximum permitted levels are for continuous consumption.

A more realistic approach to evaluating the detector's capabilities in early response to radioactive contamination is to calculate the dose resulting from the consumption of drinking water with an activity concentration corresponding to the detection limit, using the ICRP dose coefficients [18]. We assume $0.5 \mathrm{~L}(0.5 \mathrm{~kg})$ for infants and $2 \mathrm{~L}(2 \mathrm{~kg})$ for adults as a reference volume with an activity concentration corresponding to the detection limit that could be consumed before immediate protective measures should take place. The thereby calculated effective doses are displayed in Table 4. In that table, it can also be seen that the expected doses in this scenario stay below the reference level of $1 \mathrm{mSv}$ for one year [5]. This demonstrates that the integration times of $60 \mathrm{~s}$ are sufficient for application of this online detector as an early warning system in order to be able to trigger immediate protective measures.

\section{Conclusion and outlook}

We were able to successfully develop an online detector as a monitoring system for radionuclides in drinking water systems. Measurements with typical alpha, beta and gamma emitters show that the system is able to reach detection limits that correspond to doses less than $1 \mathrm{mSv}$ with sampling times that allow for immediate measures. The presented monitoring system does not offer the same detection limits as, for example, the TAWARA ${ }^{1}$ project [4] but differs from this system by, for example, refraining from lead shielding, a read out with two photomultipliers and VMEbus ${ }^{2}$ modules, making our system an easily portable stand-alone device with detection limits that still allow for the intended use as online monitor with warning system that triggers further investigation. Due to its simple setup, the prototype of our device has proven to be cost efficient and simple in handling and therefore being suitable for usage with remote monitoring and trigger signalling without displaying the full data set. It can therefore easily be used by emergency responders that might not have dedicated training in radiation detection techniques. Despite the sensitivity already being sufficient for online monitoring, an upgrade to further improve the detector system is currently being developed. In this upgrade, the detector will be equipped with a light guide to reduce the conversion factor resulting in lower detection limits. Further improvements will include a self-calibration method using a light pulser or an external radiation source, which will enable the system to be operated remotely over long times without the need of local maintenance.

Funding This work was supported by the German Federal Ministry of Education and Research [promotional reference 02NUK030] as a part of the competence network TRANSAqua [3].

Availability of data and materials The datasets generated during and/ or analysed during the current study are available from the corresponding author on reasonable request.

\section{Compliance with ethical standards}

Conflict of interest The authors declare that they have no conflict of interest.

Open Access This article is licensed under a Creative Commons Attribution 4.0 International License, which permits use, sharing, adaptation, distribution and reproduction in any medium or format, as long as you give appropriate credit to the original author(s) and the source, provide a link to the Creative Commons licence, and indicate

\footnotetext{
1 TAp WAter RAdioactivity.

${ }^{2}$ Versa Module Eurocard bus-computer bus standard.
} 
if changes were made. The images or other third party material in this article are included in the article's Creative Commons licence, unless indicated otherwise in a credit line to the material. If material is not included in the article's Creative Commons licence and your intended use is not permitted by statutory regulation or exceeds the permitted use, you will need to obtain permission directly from the copyright holder. To view a copy of this licence, visit http://creativecommons .org/licenses/by/4.0/.

\section{References}

1. United Nations Scientific Committee on the Effects of Atomic Radiation, Annex A Appendix C (Assessment of doses to the public), UNSCEAR 2013 Report Volume I (2013):5

2. Carvalho FP, Fajgelj A (2013) Radioactivity in drinking water: routine monitoring and emergency response. Water, Air, Soil Pollut 224:1597

3. TransAqua (2020). https://www.helmholtz-muenchen.de/en/ $\mathrm{irm} /$ research/working-groups/preventative-radiation-prote ction/projects/competence-network-radiation-research-kvsf/ index.html. Accessed 31 May 2020

4. Carconi P, De Felice P, Fazio A, Petrucci A, Lunardon M, Moretto S, Stevanato L, Cester D, Pastore P (2017) Characterization and calibration of a novel detection system for real time monitoring of radioactive contamination in water processed at water treatment facilities. Appl Radiat Isot 126:138-145

5. The Council of the European Union, Council Regulation (Euratom) 2016/52, Official Journal of the European Union, L 13/8 (2016)

6. Arce P et al (2014) Gamos: a framework to do Geant4 simulations in different physics fields with an user-friendly interface.
Nucl Instrum Methods Phys Res Sect A: Accel, Spectrom, Detect Assoc Equip 735:304-313

7. Mirion Technologies. https://www.mirion.com/assets/802-scint illation-detectors_DN3ra2v.pdf. Accessed 24 Jan 2021

8. Epic Crystal Co., Ltd. http://www.epic-crystal.com/others/plast ic-scintillator.html. Accessed 16 Nov 2020

9. Hamamatsu Photonics K.K. https://www.hamamatsu.com/resou rces/pdf/etd/R2154-02_TPMH1089E.pdf. Accessed 24 Jan 2021

10. Mirion Technologies. https://www.mirion.com/assets/ospre y-universal-digital-mca-tube-base-for-scintillation-spectromet ry_98mvbmW.pdf. Accessed 24 Jan 2021

11. Ultimaker BV (2020). https://ultimaker.com. Accessed 31 May 2020

12. International Organization for Standardization (2010) ISO 11929:2010

13. De Felice $P$, Jerome $S$, Petrucci A (2017) Practical implementation of ISO 11929:2010. Appl Radiat Isotop 126:256-262

14. Laboratoire National Henri Becquerel. http://www.Inhb.fr/nucle ar-data/nuclear-data-table/. Accessed 31 May 2020

15. PerkinElmer Inc. https://www.perkinelmer.com/CMSResources/ Images/44-73887SPC_TriCarb3110TRLSA.pdf. Accessed 31 May 2020

16. PerkinElmer Inc. https://www.perkinelmer.com/lab-solutions/ resources/docs/APP_Cocktails-for-Liquid-Scintillation-Count ing-011940_01.pdf. Accessed 24 Jan 2021

17. Mirion Technologies. https://www.mirion.com/assets/germa nium-detectors_XJzyVk2.pdf. Accessed 24 Jan 2021

18. ICRP (2012) Compendium of dose coefficients based on ICRP publication 60 ICRP Publication 119. Ann ICRP 41(Suppl.):1-30

Publisher's Note Springer Nature remains neutral with regard to jurisdictional claims in published maps and institutional affiliations. 